\title{
Correction to: Commodity Consistent Meta-Analysis of Wetland Values: An Illustration for Coastal Marsh Habitat
}

\author{
Hermine Vedogbeton ${ }^{1} \cdot$ Robert J. Johnston ${ }^{1,2}$ (D) \\ Published online: 12 November 2020 \\ (c) Springer Nature B.V. 2020
}

\section{Correction to: Environmental and Resource Economics (2020) 75:835-865 https://doi.org/10.1007/s10640-020-00409-0}

In the original article, the code used to estimate the meta-regression models (of WTP for coastal marsh habitat changes) erroneously included the natural log of nominal rather than real income as an independent variable. The models should have included income with all values standardized to 2016 USD. Instead, the code included the natural log of nominal income prior to adjustment into constant 2016 USD.

This error had only a minor effect on results presented in the original article. Not surprisingly, the primary effect was a change in the parameter estimate on the time-trend variable, yearindex, which was also included in the model. That is, because the difference between nominal and real income has a time trend, the effect of this error was largely captured by the parameter on the time-trend variable. Statistical significance levels and/or relative magnitudes change for a few parameters in the alternative, less commodity consistent "habitat and area" meta-regression model (e.g., riparian_marsh, habitat_fish, habitat_multiple, dichotomous). Where these changes occur, estimated significance levels typically improve ( $p$-values decrease). These changes are not material to the primary hypotheses considered by the article.

Correcting the error leads to slight improvements in model fit and benefit transfer accuracy. None of the key conclusions of the article change.

Results of the corrected data and analysis are provided in Tables 1, 2, 3, 4, 5 and 6 (corrected). These correspond to Tables 1-6 in the original article. We have updated the summary statistics in Tables 1 and 2 to reflect only the observations included in the unrestricted meta-regression models $(\mathrm{N}=133$ and 151). With these updates, there is

The original article can be found online at https://doi.org/10.1007/s10640-020-00409-0.

Robert J. Johnston

rjohnston@clarku.edu

Hermine Vedogbeton

hvedogbeton@clarku.edu

1 Department of Economics, Clark University, Worcester, MA 01610, USA

2 George Perkins Marsh Institute, Clark University, 950 Main St, Worcester, MA 01610, USA 


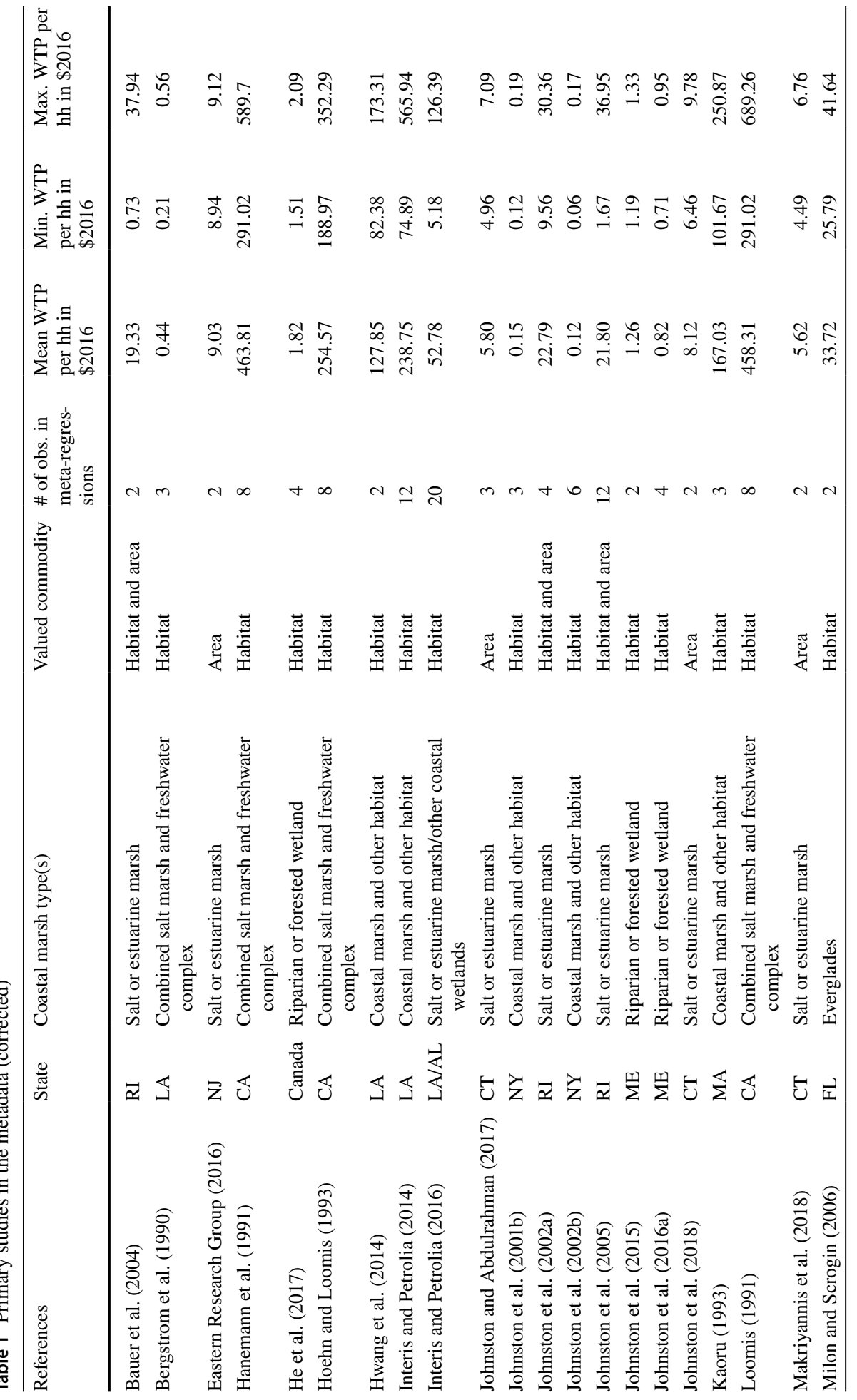




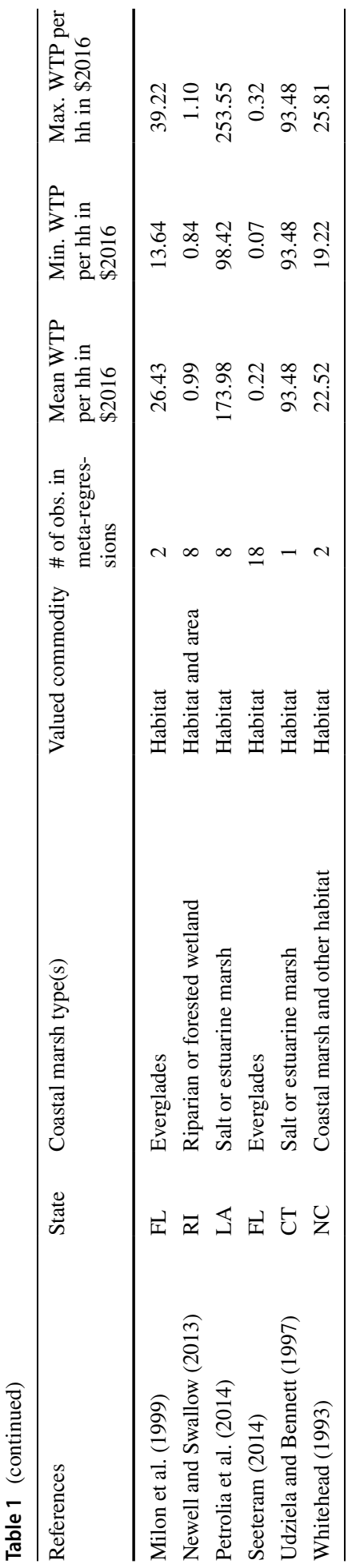




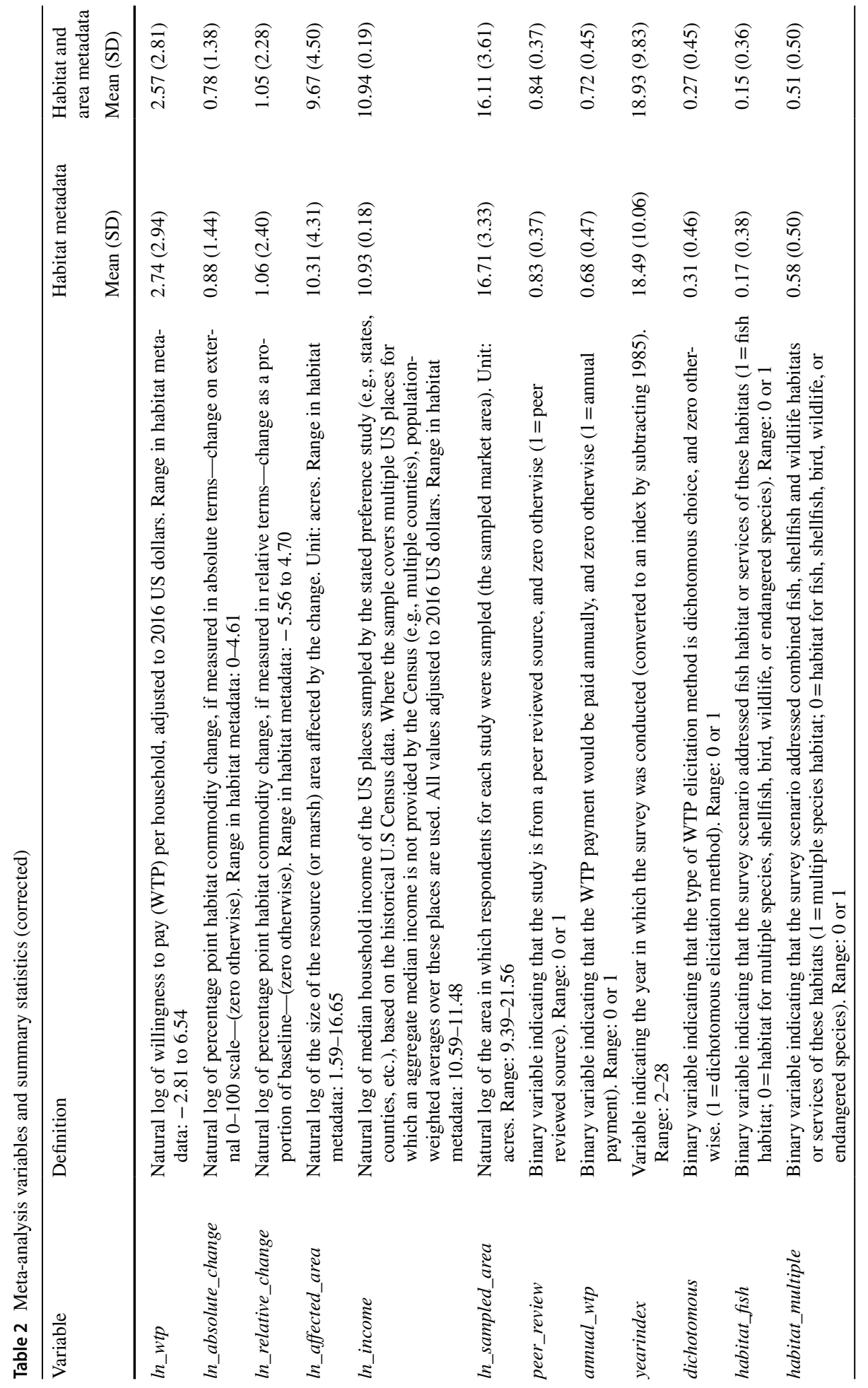




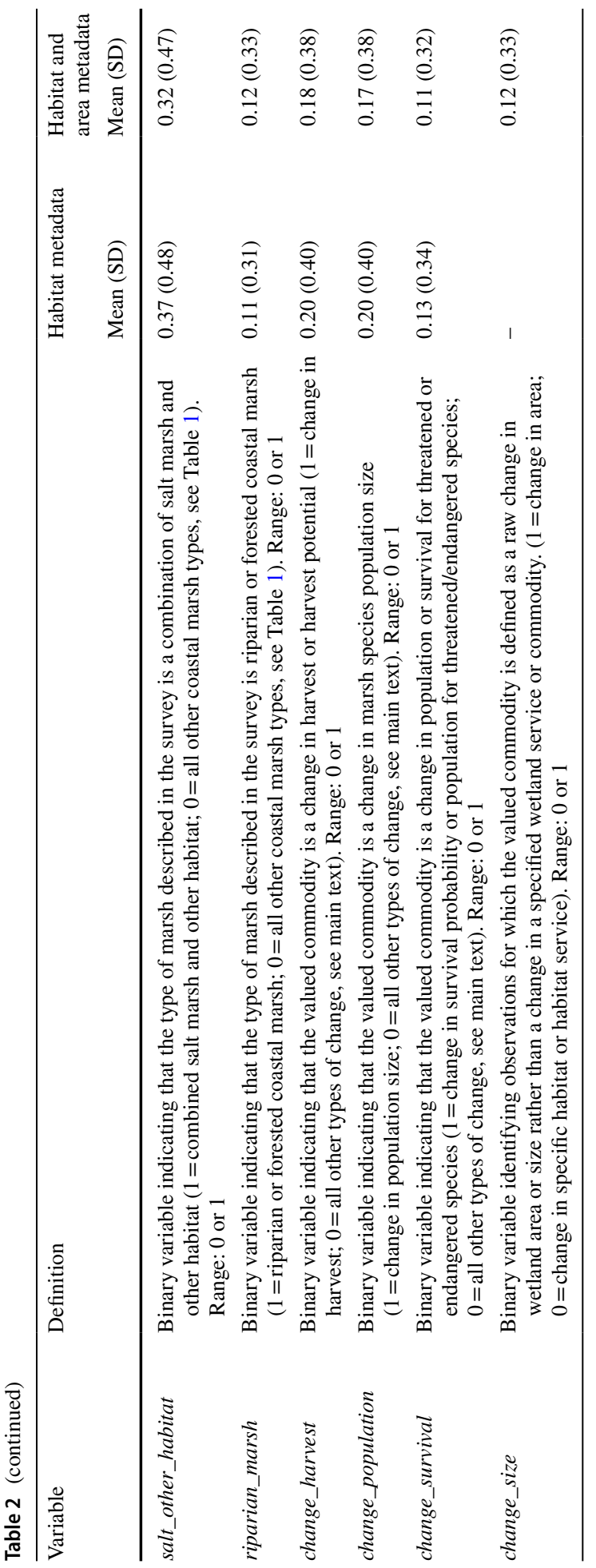


Table 3 MRM estimation results—cluster robust OLS (corrected)

\begin{tabular}{|c|c|c|c|}
\hline & $\begin{array}{l}\text { Unrestricted model: } \\
\text { habitat }\end{array}$ & Restricted model: habitat & $\begin{array}{l}\text { Unrestricted } \\
\text { model: habitat } \\
\text { and area }\end{array}$ \\
\hline ln_absolute_change & $\begin{array}{l}0.787 * * * \\
(0.099)\end{array}$ & $\begin{array}{l}0.691 * * * \\
(0.142)\end{array}$ & $\begin{array}{l}0.816^{* * * *} \\
(0.080)\end{array}$ \\
\hline In_relative_change & $\begin{array}{l}1.207 * * * \\
(0.087)\end{array}$ & $\begin{array}{l}0.960 * * * \\
(0.119)\end{array}$ & $\begin{array}{l}1.217 * * * \\
(0.062)\end{array}$ \\
\hline ln_sampled_area & $\begin{array}{l}-0.193 * * \\
(0.0809)\end{array}$ & $\begin{array}{l}0.0705 \\
(0.124)\end{array}$ & $\begin{array}{l}-0.221 * * * \\
(0.059)\end{array}$ \\
\hline ln_income & $\begin{array}{l}4.980 * * * \\
(1.043)\end{array}$ & $\begin{array}{l}4.181 * * * \\
(1.004)\end{array}$ & $\begin{array}{l}5.485 * * * \\
(0.753)\end{array}$ \\
\hline In_affected_area & $\begin{array}{l}0.157 * * \\
(0.057)\end{array}$ & $\begin{array}{l}-0.073 \\
(0.061)\end{array}$ & $\begin{array}{l}0.174 * * * \\
(0.051)\end{array}$ \\
\hline change_harvest & $\begin{array}{l}-1.309^{* * *} \\
(0.310)\end{array}$ & & $\begin{array}{l}-1.263^{* * *} \\
(0.289)\end{array}$ \\
\hline change_population & $\begin{array}{l}-1.200^{* * * *} \\
(0.272)\end{array}$ & & $\begin{array}{l}-1.321 * * * \\
(0.271)\end{array}$ \\
\hline change_survival & $\begin{array}{l}-0.627 * \\
(0.327)\end{array}$ & & $\begin{array}{l}-0.791 * * \\
(0.331)\end{array}$ \\
\hline riparian_marsh & $\begin{array}{l}-1.093 * * \\
(0.403)\end{array}$ & & $\begin{array}{l}-0.772 * * * \\
(0.267)\end{array}$ \\
\hline annual_wtp & $\begin{array}{l}-2.590 * * * \\
(0.558)\end{array}$ & $\begin{array}{l}-2.263^{* * *} \\
(0.448)\end{array}$ & $\begin{array}{l}-2.925 * * * \\
(0.500)\end{array}$ \\
\hline habitat_fish & $\begin{array}{l}-0.319 \\
(0.240)\end{array}$ & & $\begin{array}{l}-0.485^{* *} \\
(0.234)\end{array}$ \\
\hline habitat_multiple & $\begin{array}{l}-1.514 * * * \\
(0.258)\end{array}$ & & $\begin{array}{l}-1.678^{* * * *} \\
(0.240)\end{array}$ \\
\hline dichotomous & $\begin{array}{l}-0.262 \\
(0.171)\end{array}$ & & $\begin{array}{l}-0.075 \\
(0.274)\end{array}$ \\
\hline peer_review & $\begin{array}{l}0.704 \\
(0.626)\end{array}$ & & $\begin{array}{l}0.363 \\
(0.440)\end{array}$ \\
\hline yearindex & $\begin{array}{l}-0.032 \\
(0.031)\end{array}$ & $\begin{array}{l}-0.073^{* * *} \\
(0.023)\end{array}$ & $\begin{array}{l}-0.032 \\
(0.019)\end{array}$ \\
\hline salt_other_habitat & $\begin{array}{l}-0.187 \\
(0.301)\end{array}$ & & $\begin{array}{l}-0.199 \\
(0.309)\end{array}$ \\
\hline change_size & & & $\begin{array}{l}-2.193 * * * \\
(0.386)\end{array}$ \\
\hline intercept & $\begin{array}{l}-48.50^{* * *} \\
(11.21)\end{array}$ & $\begin{array}{l}-42.12 * * * \\
(10.33)\end{array}$ & $\begin{array}{l}-53.18^{* * *} \\
(7.85)\end{array}$ \\
\hline $\mathrm{N}$ & 133 & 133 & 151 \\
\hline R-sq & 0.958 & 0.912 & 0.951 \\
\hline Adj. R-sq & 0.952 & 0.907 & 0.945 \\
\hline RMSE & 0.641 & 0.897 & 0.659 \\
\hline
\end{tabular}

$* p<0.10 ; * * p<0.05 ; * * * p<0.01$ 
Table 4 Convergent validity results: absolute value percentage transfer errors (corrected)

\begin{tabular}{llll}
\hline & $\begin{array}{l}\text { Mean absolute percent } \\
\text { WTP error (\%) }\end{array}$ & $\begin{array}{l}\text { Mean absolute percent WTP } \\
\text { error }(5 \% \text { trimmed })(\%)\end{array}$ & $\begin{array}{l}\text { Median absolute } \\
\text { percent WTP error } \\
(\%)\end{array}$ \\
\hline $\begin{array}{c}\text { Unrestricted mode 1: } \\
\text { habitat }(\mathrm{N}=133)\end{array}$ & 66.51 & 50.66 & 41.49 \\
$\begin{array}{c}\text { Unrestricted model: } \\
\text { habitat and area } \\
(\mathrm{N}=151)\end{array}$ & 71.59 & 50.77 & 44.65 \\
\hline
\end{tabular}


Table 5 MRM estimation results-cluster robust WLS with publication bias test using inverse root sample size $(1 / \sqrt{n})$ (corrected)

\begin{tabular}{|c|c|}
\hline & $\begin{array}{l}\text { Unrestricted } \\
\text { model: } \\
\text { habitat }\end{array}$ \\
\hline $1 / \sqrt{n}$ & $\begin{array}{l}7.353 \\
(15.930)\end{array}$ \\
\hline ln_absolute_change & $\begin{array}{l}0.655^{* * * *} \\
(0.160)\end{array}$ \\
\hline In_relative_change & $\begin{array}{l}1.149 * * * \\
(0.114)\end{array}$ \\
\hline ln_sampled_area & $\begin{array}{l}-0.126 \\
(0.140)\end{array}$ \\
\hline In_income & $\begin{array}{l}4.419 * * * \\
(1.372)\end{array}$ \\
\hline In_affected_area & $\begin{array}{l}0.094 \\
(0.091)\end{array}$ \\
\hline change_harvest & $\begin{array}{l}-1.702^{* * * *} \\
(0.267)\end{array}$ \\
\hline change_population & $\begin{array}{l}-1.174 * * * \\
(0.274)\end{array}$ \\
\hline change_survival & $\begin{array}{l}-0.510 \\
(0.383)\end{array}$ \\
\hline riparian_marsh & $\begin{array}{l}-0.873^{*} \\
(0.423)\end{array}$ \\
\hline annual_wtp & $\begin{array}{l}-2.933 * * * \\
(0.444)\end{array}$ \\
\hline habitat_fish & $\begin{array}{l}-0.130 \\
(0.262)\end{array}$ \\
\hline habitat_multiple & $\begin{array}{l}-1.298^{* * * *} \\
(0.303)\end{array}$ \\
\hline dichotomous & $\begin{array}{l}-0.284^{*} \\
(0.148)\end{array}$ \\
\hline peer_review & $\begin{array}{l}0.489 \\
(0.689)\end{array}$ \\
\hline yearindex & $\begin{array}{l}-0.046 \\
(0.029)\end{array}$ \\
\hline salt_other_habitat & $\begin{array}{l}-0.052 \\
(0.260)\end{array}$ \\
\hline intercept & $\begin{array}{l}-42.40 * * * \\
(13.79)\end{array}$ \\
\hline $\mathrm{N}$ & 133 \\
\hline R-sq & 0.954 \\
\hline RMSE & 0.616 \\
\hline
\end{tabular}

Observations are weighted by sample size $(n)$ ${ }^{*} p<0.10 ; * * p<0.05 ; * * * p<0.01$ 
Table 6 MRM2 estimation results-dependent variable defined as the natural log of WTP per percentage point habitat change (corrected)

\begin{tabular}{|c|c|}
\hline & $\begin{array}{l}\text { Unrestricted } \\
\text { model: } \\
\text { habitat }\end{array}$ \\
\hline ln_absolute_change & $\begin{array}{l}-0.213 * * \\
(0.099)\end{array}$ \\
\hline In_relative_change & $\begin{array}{l}0.207 * * \\
(0.087)\end{array}$ \\
\hline ln_sampled_area & $\begin{array}{l}-0.193 * * \\
(0.081)\end{array}$ \\
\hline ln_income & $\begin{array}{l}4.980 * * * \\
(1.043)\end{array}$ \\
\hline In_affected_area & $\begin{array}{l}0.157 * * \\
(0.057)\end{array}$ \\
\hline change_harvest & $\begin{array}{l}-1.309 * * * \\
(0.310)\end{array}$ \\
\hline change_population & $\begin{array}{l}-1.200 * * * \\
(0.272)\end{array}$ \\
\hline change_survival & $\begin{array}{l}-0.627^{*} \\
(0.327)\end{array}$ \\
\hline riparian_marsh & $\begin{array}{l}-1.093 * * \\
(0.403)\end{array}$ \\
\hline annual_wtp & $\begin{array}{l}-2.590 * * * \\
(0.558)\end{array}$ \\
\hline habitat_fish & $\begin{array}{l}-0.319 \\
(0.240)\end{array}$ \\
\hline habitat_multiple & $\begin{array}{l}-1.514 * * * \\
(0.258)\end{array}$ \\
\hline dichotomous & $\begin{array}{l}-0.262 \\
(0.171)\end{array}$ \\
\hline peer_review & $\begin{array}{l}0.704 \\
(0.626)\end{array}$ \\
\hline yearindex & $\begin{array}{l}-0.032 \\
(0.031)\end{array}$ \\
\hline salt_other_habitat & $\begin{array}{l}-0.187 \\
(0.301)\end{array}$ \\
\hline intercept & $\begin{array}{l}-48.50 * * * \\
(11.21)\end{array}$ \\
\hline $\mathrm{N}$ & 133 \\
\hline R-sq & 0.858 \\
\hline RMSE & 0.641 \\
\hline
\end{tabular}

$*_{p}<0.10 ; * * p<0.05 ; * * * p<0.01$

now a precise match between the observations included in the meta-regression models (Tables 3, 4, 5, 6) and those used for data summary statistics (Tables 1 and 2).

This correction stands to correct the original article. 
Publisher's Note Springer Nature remains neutral with regard to jurisdictional claims in published maps and institutional affiliations. 